\title{
Thermodynamics of a heavy-ion-irradiated superconductor: The zero-field transition
}

\author{
C. J. van der Beek \\ Laboratoire des Solides Irradiés, CNRS-UMR 7642 \& CEA/DSM/DRECAM, Ecole Polytechnique, 91128 Palaiseau cedex, France
}

Thierry Klein and René Brusetti

Institut Néel, Boîte Postale 166, 38042 Grenoble cedex 9, France

Christophe Marcenat

Département de Recherche Fondamentale sur la Matière Condensée/SPSMS/LaTEQS,

Commissariat à l'Energie Atomique, 17 Avenue des Martyrs, 38054 Grenoble cedex 9, France

Mats Wallin

Department of Theoretical Physics, KTH, AlbaNova University Center, SE-106 91 Stockholm, Sweden

S. Teitel

Department of Physics and Astronomy, University of Rochester, Rochester, New York 14627, USA

Hans Weber

Division of Physics, Luleå University of Technology, SE-971 87 Luleå, Sweden

(Received 29 January 2007; published 9 March 2007)

\begin{abstract}
Specific heat measurements show that the introduction of amorphous columnar defects considerably affects the transition from the normal to the superconducting state in zero magnetic field. Experimental results are compared to numerical simulations of the three-dimensional $X Y$ model for both the pure system and the system containing random columnar disorder. The numerics reproduce the salient features of experiment, showing in particular that the specific heat peak changes from cusplike to smoothly rounded when columnar defects are added. By considering the specific heat critical exponent $\alpha$, we argue that such behavior is consistent with recent numerical work [A. Vestergren et al., Phys. Rev. B 70, 054508 (2004)] showing that the introduction of columnar defects changes the universality class of the transition.
\end{abstract}

DOI: $10.1103 /$ PhysRevB.75.100501

PACS number(s): 74.25.Bt, 74.40.+k, 68.35.Rh

An enormous amount of attention has been paid in recent years to the effect of amorphous columnar defects on the superconducting transition in a magnetic field. ${ }^{1-7}$ Much less work has been done on the transition in zero magnetic field, the only report to our knowledge being the measurement of the microwave conductance transverse to the columnar defects. ${ }^{7}$ Nevertheless, the transition in zero magnetic field merits attention in its own right. ${ }^{8}$ It is expected that disorder will be a relevant perturbation and change the universality class of a phase transition whenever $2-d^{*} \nu>0$ (modified Harris criterion ${ }^{9}$ ). Here $d^{*}$ is the number of dimensions in which the system is disordered and $\nu$ is the usual correlation length critical exponent. In the absence of columnar defects, we expect the superconducting phase transition to fall in the universality class of the three-dimensional (3D) $X Y$ model, so that $\nu=0.6717(1) .{ }^{10}$ In the case of random point disorder, $d^{*}=3$, so that $2-d^{*} \nu \approx-0.015<0$, and the disorder is irrelevant. In the case of columnar disorder, however, $d^{*}=2$, so that $2-d^{*} \nu \approx 0.66>0$, and disorder should be relevant and drive the system to a new universality class. Note that the stability of the new disordered critical point with respect to the modified Harris criterion requires that the new correlation length critical exponent satisfy $\nu>1 .^{8}$ Recent simulations of a columnar-disordered $X Y$ model in Ref. 8 supported such expectations, finding a phase transition with anisotropic scaling and a value for the critical exponent $\nu \approx 1.2$.

It is the purpose of this Rapid Communication to test these ideas on a real superconducting system. For this, we have chosen to measure the specific heat of optimally doped, single-crystalline $\mathrm{YBa}_{2} \mathrm{Cu}_{3} \mathrm{O}_{7-\delta}$, both without and with columnar defects. It has been shown previously ${ }^{11,12}$ that the specific heat at the superconducting transition of pristine $\mathrm{YBa}_{2} \mathrm{Cu}_{3} \mathrm{O}_{7-\delta}$ is consistent with that of the $3 \mathrm{D} X Y$ model, with a small negative specific heat exponent $\alpha \approx-0.015 .{ }^{10} \mathrm{It}$ turns out that this exponent is considerably modified by the introduction of the columnar defects.

Experiments were done on two $\mathrm{YBa}_{2} \mathrm{Cu}_{3} \mathrm{O}_{7-\delta}$ single crystals, cut from the same piece. The original crystal was grown by the flux method in $\mathrm{Au}$ crucibles and subsequently annealed in oxygen in Pt tubes. ${ }^{13}$ One crystal, which contained a single family of twin boundaries separated by a distance of approximately $10 \mu \mathrm{m}$, was irradiated with $5.8-\mathrm{GeV} \mathrm{Pb}$ ions at the Grand Accélérateur National d'Ions Lourds (GANIL) in Caen, France, to a fluence of $1 \times 10^{11}$ ions $\mathrm{cm}^{-2}$. The ion beam was directed parallel to the $c$ axis; each ion impact created an amorphous columnar track of radius $\approx 3.5 \mathrm{~nm}$. The second, untwinned crystal, was not irradiated, but kept as the pristine reference sample. Specific heat measurements have been performed in the absence of an applied magnetic field, using the same measurement technique employed in Ref. 14.

In Fig. 1(a) we show our raw data for the specific heat of both the pristine and irradiated crystals. The temperature axis has been scaled by the value of the temperature $T_{\text {peak }}$ at the 

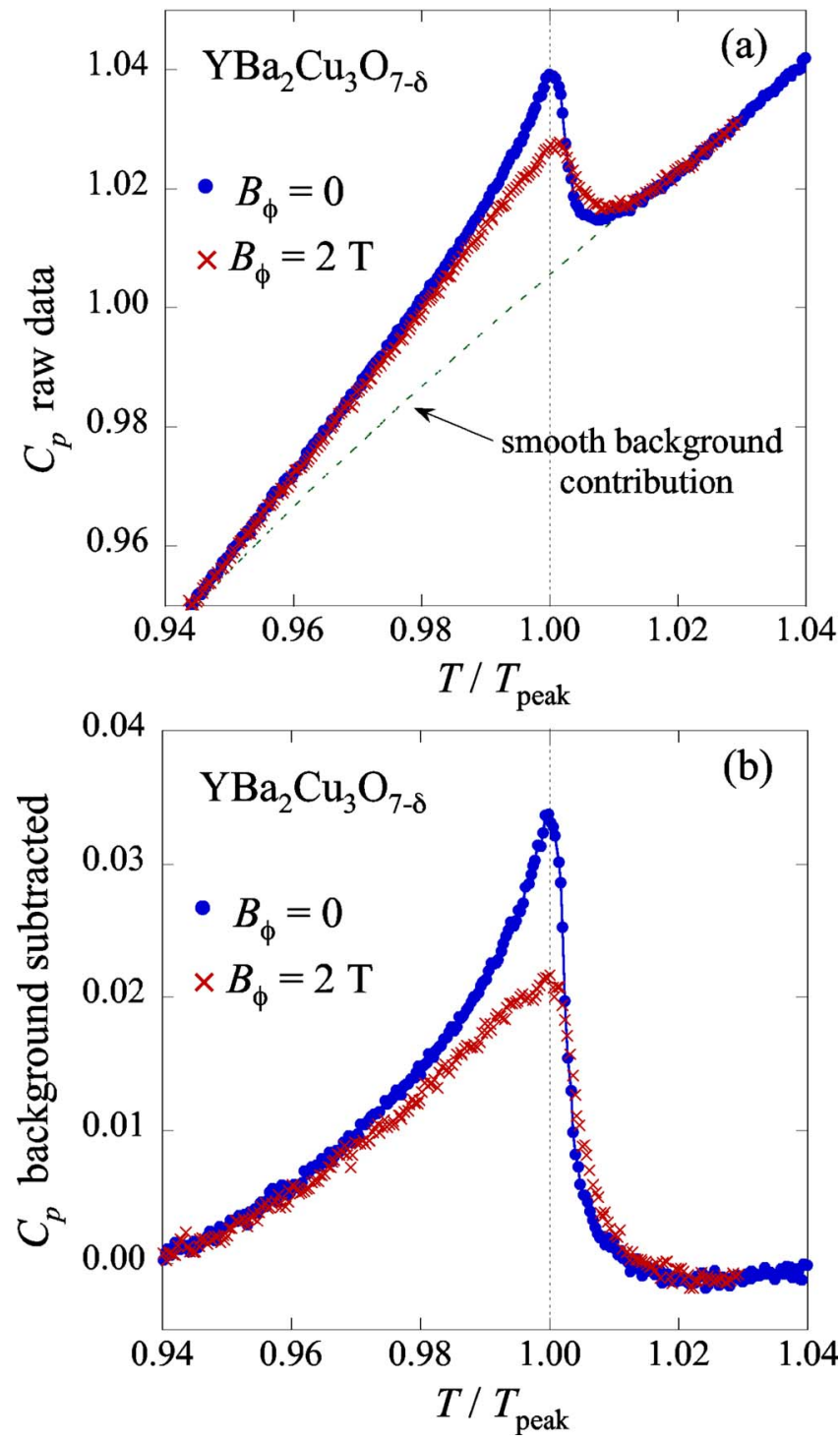

FIG. 1. (Color online) (a) Raw specific heat data (in arbitrary units) taken on a pristine $\mathrm{YBa}_{2} \mathrm{Cu}_{3} \mathrm{O}_{7-\delta}$ crystal (O) and on a crystal with a columnar defect density $n_{d}=1 \times 10^{11} \mathrm{~cm}^{-2}$-i.e., a matching field $B_{\phi}=\Phi_{0} n_{d}=2 \mathrm{~T}(\times)$. In order to compare the two data sets, the temperature has been rescaled by the value $T_{\text {peak }}$ at which the specific heat peak occurs. The dashed line represents the smooth background contribution to the specific heat, which is estimated using a third-order polynomial fit. This background is subtracted in (b) in order to emphasize the shape of the specific heat peak due to the superconducting transition.

specific heat peak, in order to better compare the two samples $\left(T_{\text {peak }}=93.1 \mathrm{~K}\right.$ for the pristine sample, while $T_{\text {peak }}$ $=92.1 \mathrm{~K}$ for the irradiated sample). We see clear specific heat anomalies, signatures of the superconducting phase transition, superimposed on a smooth increasing background. In the pristine sample, the amplitude of the specific heat anomaly was of the order of $4 \%$ of the total specific heat, attesting to its very high quality. Fitting the smooth background to a cubic polynomial [dashed line in Fig. 1(a)] we subtract this background from the data in Fig. 1(b), in order to better emphasize the shape of the anomaly. For the pristine sample, we see the typical " $\lambda$ "-cusp shape expected for the
$H=0$ superconducting transition in the presence of strong thermal fluctuations. The introduction of the amorphous columnar defects reduces the absolute temperature at which the specific heat maximum occurs. Note that a lowering of the critical temperature after heavy-ion irradiation may occur as a result of "self-doping" of the intercolumn space by $\mathrm{O}$ ions expelled from the tracks; ${ }^{15,16}$ however, no such effect was reported for $\mathrm{YBa}_{2} \mathrm{Cu}_{3} \mathrm{O}_{7-\delta}$. Another possibility is that the columns reduce the average $T_{c}$ at which long-range superconducting order can set in. ${ }^{17,18}$ The resulting specific heat curve after heavy-ion bombardment shows notable differences with respect to the curve before irradiation. Most specifically, we find that the shape of the maximum is now smoothly rounded rather than the sharp cusp seen in the pristine sample. Following the suggestion ${ }^{8}$ that the introduction of columnar defects changes the universality class of the superconducting transition in zero field, we propose that the associated change of critical exponents is at the origin of the markedly different shape of the specific heat peak before and after irradiation.

The presence of columnar defects implies that, even in zero magnetic field, critical scaling of physical quantities may be anisotropic: the correlation length parallel to the columns, $\xi_{z}$, diverges as a different power of the reduced temperature $t \equiv\left(T-T_{c}\right) / T_{c}$ than the correlation length in the transverse direction $\xi_{\perp}$. This defines the anisotropy exponent $\zeta$

$$
\xi_{z} \sim \xi_{\perp}^{\zeta}
$$

Defining the correlation length exponent $\nu$ in the usual way, $\xi_{\perp} \sim|t|^{-\nu}$, the singular part of the free energy density will scale as

$$
f(T) \sim\left(\xi_{z} \xi_{\perp}^{2}\right)^{-1} \sim \xi_{\perp}^{-2-\zeta} \sim|t|^{\nu(2+\zeta)} .
$$

As a consequence, the specific heat per unit volume, $c$, will scale as

$$
c \sim \frac{\partial^{2} f}{\partial t^{2}} \sim|t|^{\nu(2+\zeta)-2} \equiv|t|^{-\alpha} .
$$

Thus, the specific heat exponent in the anisotropic case is $\alpha=2-\nu(2+\zeta)$. For the pure system-i.e., a superconductor without columnar defects - the anisotropy exponent $\zeta=1$ and $\nu \gtrsim \frac{2}{3}$. The specific heat exponent $\alpha$ is small and slightly negative. In contrast, the calculation for the $X Y$ model of a superconductor with columnar defects of Ref. 8 found values $\zeta \approx 1.3$ and $\nu \approx 1.2$. In this case, $\alpha \approx-2.0$ and is thus much more strongly negative.

Because $\alpha$ is negative for both cases, the specific heat does not diverge at the transition. However, an interesting difference is seen if we consider the temperature derivative of the specific heat,

$$
\frac{d c}{d t} \sim|t|^{-\alpha-1}
$$

For the pure superconductor, $-\alpha-1 \approx-0.985$ is negative. Therefore, the slope of the specific heat diverges at $T_{c}$, giving rise to the familiar cusp observed in Fig. 1 for the pristine sample. For the superconductor with columnar defects, how- 
ever, $-\alpha-1 \approx 1.0$ is positive, so the slope of $c$ does not diverge. There is no sharp cusp, as is indeed observed experimentally. ${ }^{19}$

The above discussion considers only the singular part of the specific heat that comes from the large length scale critical fluctuations. It disregards the smooth nonsingular contribution that comes from the noncritical short-length-scale fluctuations. Most likely, this nonsingular part of the specific heat has some nonzero temperature derivative at $T_{c}$. For the unirradiated superconductor, this temperature derivative is much smaller than the diverging slope of the singular part and can therefore be disregarded. Thus, the nonsingular contribution is unlikely to affect the shape of the $c$ curve very much. In the presence of columnar defects, however, the slope of the singular part vanishes at $T_{c}$. Then, the temperature derivative of the specific heat at $T_{c}$ is determined by the nonsingular part. Since the slope of $c$ is nonzero and smooth at $T_{c}$, the maximum of $c$ is no longer located at the critical temperature and the shape of the peak is now a smoothly curved maximum. As we discuss below, this feature is also found in the experimental data.

To illustrate the comparison further, we have carried out Monte Carlo simulations to numerically compute $c$ for the pure system, and also for the system with columnar disorder, using the same $X Y$ model and random distribution for the columnar disorder as defined in Sec. II A of Ref. 8. For the pure case, we use a system size of $40^{3}$ grid sites. For the case with columnar disorder we used two different system sizes. Taking $L=L_{x}=L_{y}$ as the system length transverse to the direction of the columnar defects and using $L_{z}=0.5 L^{\zeta}$ ( $\zeta$ $=1.3$ ) consistent with anisotropic scaling, we considered sizes $L=20$, averaged over 125 different realizations of the random disorder, and $L=40$, averaged over 66 different realizations of disorder. Our results are shown in Fig. 2. It is clearly seen that the pure case displays a peak with the familiar " $\lambda$ " cusp, whereas the case with columnar disorder displays a smoothly rounded peak. We observe essentially no size dependence in our results for the case with columnar disorder. Our numerical results thus reproduce the main qualitative features found experimentally for single-crystalline $\mathrm{YBa}_{2} \mathrm{Cu}_{3} \mathrm{O}_{7-\delta}$. The difference in details between the experimental and model curves for the case with columnar defects most likely results from the simplicity of the numerical model as well as differences in the effective strength of the disorder. For the simulations, a particularly strong disorder was chosen so as to reach the asymptotic limit even with the small system sizes under scrutiny. Presumably, this explains why the model curve is even rounder than the experimental one.

Another feature of the numerical results is that, in agreement with the argument above, the system with columnar disorder is found to have a specific heat peak that lies below the critical temperature, with $\left(T_{c}-T_{\text {peak }}\right) / T_{c}=0.07$. In order to compare this result with experiment, the critical temperature of the $\mathrm{YBa}_{2} \mathrm{Cu}_{3} \mathrm{O}_{7-\delta}$ crystals is deduced from the functional dependence of the thermodynamic properties of the superconductor on a relevant "scaling" parameter. In Ref. 20, it was shown that, for magnetic fields $B$ larger than $1 \mathrm{~T}$, the field and temperature dependence of the magnetization of the crystals under study can be described by a unique [Ginzburg-

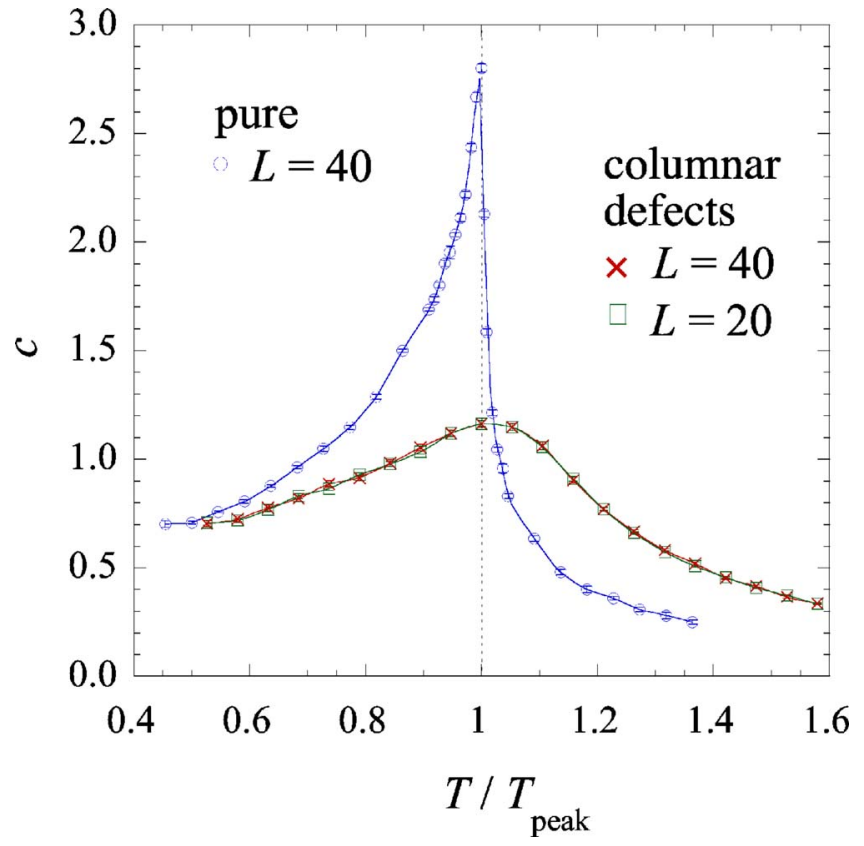

FIG. 2. (Color online) Monte Carlo calculation of the specific heat using the 3D $X Y$ model for a pure (pristine) superconductor ( $\bigcirc)$ and a superconductor containing columnar disorder. For the pure case, a system of size $40^{3}$ was used, while for the disordered case the calculation was done for two system sizes, with $L=40$ $(\times)$ and $L=20(\square)$ grid points in the direction perpendicular to the anisotropy axis; parallel to the anisotropy axis, $L_{z}=0.5 L^{1.3}$. The temperature axis has been rescaled by the value $T_{\text {peak }}$ at which the specific heat peak occurs.

Landau lowest Landau level (GL-LLL)] relation $M /(\vartheta b)^{2 / 3}$ $\propto F(Q)$, with $Q \equiv(1-b)\left(1-\vartheta^{2}\right)^{1 / 3}(\vartheta b)^{-2 / 3}$, where $\vartheta \equiv T / T_{c}$ and $b \equiv B / B_{c 2}(T)$; here, $T_{c}$ is the zero-field critical temperature and $B_{c 2}(T)$ the upper critical field. For the unirradiated crystal, this relation gives a critical temperature $T_{c}=93.1 \mathrm{~K}$, which coincides to within $0.1 \mathrm{~K}$ with the position of the zero-field specific heat maximum. For the irradiated crystal, a similar analysis gives, within the accuracy of the fit, the same critical temperature $T_{c}=93.1 \mathrm{~K}$, which now lies above the observed specific heat maximum at $92.1 \mathrm{~K}$. The experimental data thus show a separation $\left(T_{c}-T_{\text {peak }}\right) / T_{c}=0.01$, in the same direction as the numerical results, but smaller in magnitude, again presumably due to the larger disorder strength used in the numerical model calculations.

In summary, we have measured the specific heat of pristine and heavy-ion-irradiated single-crystalline $\mathrm{YBa}_{2} \mathrm{Cu}_{3} \mathrm{O}_{7-\delta}$ in zero applied magnetic field. The results were compared to Monte Carlo simulations of the 3D XY model for both the pure case and the case with columnar disorder. Both experiment and numerics show a drastic influence of the columnar defects on the shape of the specific heat anomaly at the superconducting-to-normal transition. The overall features of the specific heat anomaly are well explained by the critical exponents obtained from the numerical calculations, suggesting that the introduction of the columnar defects does indeed change the universality class of the transition. 
We are very grateful to $\mathrm{F}$. Holtzberg for providing the $\mathrm{YBa}_{2} \mathrm{Cu}_{3} \mathrm{O}_{7-\delta}$ crystals. The work of S.T. is supported by U.S. Department of Energy Grant No. DE-FG02-06ER46298. The work of M.W. is supported by the Swedish Research Council
(VR) and the Swedish National Infrastructure for Computing (SNIC). H.W. acknowledges support from Swedish Research Council Contract No. 621-2001-2545.
${ }^{1}$ D. R. Nelson and V. M. Vinokur, Phys. Rev. Lett. 68, 2398 (1992); Phys. Rev. B 48, 13060 (1993).

${ }^{2}$ G. Blatter, M. V. Feigel'man, V. B. Geshkenbein, A. I. Larkin, and V. M. Vinokur, Rev. Mod. Phys. 66, 1125 (1994), and references therein.

${ }^{3}$ M. V. Feigel'man, V. B. Geshkenbein, L. B. Ioffe, and A. I. Larkin, Phys. Rev. B 48, 16641 (1993).

${ }^{4}$ L. Krusin-Elbaum, L. Civale, G. Blatter, A. D. Marwick, F. Holtzberg, and C. Feild, Phys. Rev. Lett. 72, 1914 (1994); A. V. Samoilov and M. Konczykowski, ibid. 75, 186 (1995); L. Krusin-Elbaum, G. Blatter, and L. Civale, ibid. 75, 187 (1995).

${ }^{5}$ A. I. Larkin and V. M. Vinokur, Phys. Rev. Lett. 75, 4666 (1995).

${ }^{6}$ A. V. Samoilov, M. V. Feigel'man, M. Konczykowski, and F. Holtzberg, Phys. Rev. Lett. 76, 2798 (1996); M. Konczykowski and A. V. Samoilov, ibid. 78, 1830 (1997).

${ }^{7}$ G. Nakielski, A. Rickertsen, T. Steinborn, J. Wiesner, G. Wirth, A. G. M. Jansen, and J. Kötzler, Phys. Rev. Lett. 76, 2567 (1996); J. Kötzler, Nucleation of Stable Superconductivity in YBCO-Films, in Advances in Solid State Physics 39, edited by B. Kramer (Vieweg \& Sohn, Braunschweig, 1999), p. 371 (cond-mat/9904279).

${ }^{8}$ A. Vestergren, M. Wallin, S. Teitel, and H. Weber, Phys. Rev. B 70, 054508 (2004).

${ }^{9}$ M. Wallin, E. S. Sørensen, S. M. Girvin, and A. P. Young, Phys. Rev. B 49, 12115 (1994).

${ }^{10}$ M. Campostrini, M. Hasenbusch, A. Pelissetto, and E. Vicari, Phys. Rev. B 74, 144506 (2006).
${ }^{11}$ N. Overend, M. A. Howson, and I. D. Lawrie, Phys. Rev. Lett. 72, 3238 (1994).

${ }^{12}$ C. Marcenat, R. Calemczuk, and A. Carrington, in Coherence in High Temperature Superconductors, edited by G. Deutscher and A. Revcolevschi (World Scientific, Singapore, 1996), p. 101.

${ }^{13}$ F. Holtzberg and C. Feild, Eur. J. Solid State Inorg. Chem. 27, 107 (1990).

${ }^{14}$ C. Marcenat, S. Blanchard, J. Marcus, L. M. Paulius, C. J. van der Beek, M. Konczykowski, and T. Klein, Phys. Rev. Lett. 90, 037004 (2003).

${ }^{15}$ A. Pomar, Z. Konstantinovic, L. Martel, Z. Z. Li, and H. Raffy, Phys. Rev. Lett. 85, 2809 (2000).

${ }^{16}$ M. Li, C. J. van der Beek, M. Konczykowski, H. W. Zandbergen, and P. H. Kes, Phys. Rev. B 66, 014535 (2002).

${ }^{17}$ C. J. van der Beek, M. Konczykowski, T. W. Li, P. H. Kes, and W. Benoit, Phys. Rev. B 54, R792 (1996).

${ }^{18}$ G. M. Braverman, S. A. Gredeskul, and Y. Avishai, Phys. Rev. B 65, 054512 (2002).

${ }^{19}$ Note that the specific heat derivative in general will have the form $d c / d t=A_{ \pm}|t|^{-\alpha-1}$, where the coefficient $A_{+}$for $t>0$ is different from the coefficient $A_{-}$for $t<0$. Thus, although for $\alpha \approx-2.0$ the first temperature derivative of $c$ is continuous at $T_{c}$, the second temperature derivative should jump discontinuously at $T_{c}$.

${ }^{20}$ C. J. van der Beek, M. Konczykowski, L. Fruchter, R. Brusetti, Thierry Klein, Jacques Marcus, and C. Marcenat, Phys. Rev. B 72, 214504 (2005). 